\title{
Decreased Maximal Tongue Protrusion Length May Predict the Presence of Dysphagia in Stroke Patients
}

\author{
Hyunchul Cho, $\mathrm{MD}^{1}$, Jeong Se Noh, $\mathrm{MD}^{1}$, Junwon Park, $\mathrm{MD}^{1}$, Changwook Park, $\mathrm{MD}^{1}$, No Dam Park, $\mathrm{MD}^{2}$, \\ Jun Young Ahn, MD $^{3}$, Ji Woong Park, MD, hhD $^{1}$, Yoon-Hee Choi, MD, PhD ${ }^{1}$, Seong-Min Chun, MD, MS ${ }^{1}$ \\ ${ }^{1}$ Department of Physical Medicine and Rehabilitation, Soonchunhyang University Seoul Hospital, Seoul; \\ ${ }^{2}$ Department of Physical Medicine and Rehabilitation, Soonchunhyang University Cheonan Hospital, Cheonan; \\ ${ }^{3}$ Department of Physical Medicine and Rehabilitation, Soonchunhyang University Bucheon Hospital, Bucheon, Korea
}

Objective To investigate the relationship between maximal tongue protrusion length (MTPL) and dysphagia in post-stroke patients.

Methods Free tongue length (FTL) was measured using the quick tongue-tie assessment tool and MTPL was measured using a transparent plastic ruler in 47 post-stroke patients. The MTPL-to-FTL (RMF) ratio was calculated. Swallowing function in all patients was evaluated via videofluoroscopic swallowing study (VFSS), PenetrationAspiration Scale (PAS), Functional Oral Intake Scale (FOIS), and Videofluoroscopic Dysphagia Scale (VDS).

Results The MTPL and RMF values were significantly higher in the non-aspirator group than in the aspirator group (MTPL, $\mathrm{p}=0.0049$; RMF, $\mathrm{p}<0.001$ ). MTPL and RMF showed significant correlations with PAS, FOIS and VDS scores. The cut-off value in RMF for the prediction of aspiration was 1.56 , with a sensitivity of $84 \%$ and a specificity of $86 \%$. Conclusion There is a relationship between MTPL and dysphagia in post-stroke patients. MTPL and RMF can be useful for detecting aspiration in post-stroke patients.

Keywords Deglutition disorders, Stroke, Videofluoroscopic swallowing study

\section{INTRODUCTION}

Dysphagia is one of the most common complications in patients with stroke. Up to $65 \%$ of such patients develop post-stroke dysphagia (PSD) [1]. PSD is not only associated with quality of life (QOL) in stroke patients, but also with complications such as aspiration pneumonia, malnutrition, and dehydration. It is also linked to death and

\footnotetext{
Received July 2, 2021; Revised August 12, 2021; Accepted September 13, 2021; Published online December 31, 2021 Corresponding author: Seong-Min Chun

Department of Physical Medicine and Rehabilitation, Soonchunhyang University Seoul Hospital, 59, Daesagwan-ro, Yongsan-gu, Seoul 04401, Korea. Tel: +82-2-709-9086, Fax: +82-2-710-3088, E-mail: neokai7@naver.com

ORCID: Hyunchul Cho (https://orcid.org/0000-0001-5103-0507); Jeong Se Noh (https://orcid.org/0000-0003-4529-3225); Junwon Park (https://orcid. org/0000-0003-1680-0048); Changwook Park (https://orcid.org/0000-0002-8795-7897); No Dam Park (https://orcid.org/0000-0002-2871-0202); Jun Young Ahn (https://orcid.org/0000-0003-4340-2774); Ji Woong Park (https://orcid.org/0000-0001-7987-3581); Yoon-Hee Choi (https://orcid.org/00000001-6651-7908); Seong-Min Chun (https://orcid.org/0000-0002-2174-8742).
}

(c) This is an open-access article distributed under the terms of the Creative Commons Attribution Non-Commercial License (http://creativecommons.org/ licenses/by-nc/4.0) which permits unrestricted noncommercial use, distribution, and reproduction in any medium, provided the original work is properly cited. Copyright ( 2021 by Korean Academy of Rehabilitation Medicine 
long-term negative prognosis [2]. In the presence of aspiration, the risk of aspiration pneumonia is approximately seven-fold higher than in the group without aspiration. The presence or absence of aspiration is, therefore, an important risk factor associated with the development of pneumonia in stroke patients, underscoring the need for early diagnosis of dysphagia and appropriate management.

Patients afflicted with stroke exhibit delayed swallowing reflexes and impaired pharyngeal contractions due to motor weakness and volitional motor control. Although the risk of dysphagia also depends on the sites of the lesion $[3,4]$, it is important to measure key indicators related to the tongue and suprahyoid muscles.

The suprahyoid muscle plays an important role in airway protection and the opening of the upper esophageal sphincter, via anterior and superior displacement of the hyoid bone in the pharyngeal phase. Thus, it is a major target in the rehabilitation of patients with dysphagia [58]. Part of the suprahyoid muscle is attached to the mandible, forming the floor of the oral cavity, and together with the intrinsic and extrinsic tongue muscles participates involuntary tongue protrusion [9-11]. The tongue plays an important role in normal swallowing function by facilitating bolus transport in the oral phase, but also mediates laryngeal elevation and closure in the pharyngeal phase [12].

Many studies have revealed the link between PSD and one of the tongue-related indicators [13-15]. Studies have reported an increase in tongue pressure and suprahyoid activity with increased maximal tongue protrusion length (MTPL) [13,16-18]. Recent studies suggest a correlation between MTPL and tongue strength and MTPL and suprahyoid activity $[16,18,19]$. We, therefore, hypothesized that MTPL, along with tongue pressure and suprahyoid muscle activity, are also important indicators in evaluating dysphagia. However, the relationship between MTPL and PSD has yet to be elucidated.

Bedside screening tests for patients with acute stroke include the Gugging Swallowing Screen (GUSS), Johns Hopkins Hospital Brain Rescue Unit Modified 3 oz Swallow Screen, and the water swallow test [20-22]. However, these screening tests are designed to evaluate signs of aspiration such as reflex cough, and almost $40 \%$ of them fail to identify PSD because they are unable to exclude silent aspiration [23].
The purpose of this study was to investigate the relationship between MTPL and the presence of dysphagia in post-stroke patients. We set a cut-off value for a second goal, which was used as a screening test to determine whether or not the tongue length reflects PSD.

\section{MATERIALS AND METHODS}

This study was designed as a prospective cross-sectional study.

\section{Participants}

Patients with stroke undergoing rehabilitation at Department of Physical Medicine and Rehabilitation at Soonchunhyang University Seoul Hospital were recruited from April, 2020 to March, 2021. The study was approved by the Institutional Review Board of Soonchunhyang University Seoul Hospital (No. SCHUH 2020-10-023). All participants were evaluated via tongue length examination and videofluoroscopic swallowing study (VFSS) in the study.

Inclusion criteria were (1) stroke, confirmed by magnetic resonance imaging and/or computed tomography; (2) symptoms and/or signs of dysphagia after stroke (choking, cough, wet voice after swallowing or a history of aspiration pneumonia); (3) evaluation by VFSS; and (4) ability to follow the physician's orders.

Exclusion criteria were (1) difficulty understanding the examination due to cognitive impairment, psychiatric disorders or hearing loss; (2) refusal to participate in the study; (3) tongue deformity due to medical disease, or resection of the tongue; and (4) deformity of the maxillary central incisor due to disease or trauma.

Before commencing the study, the purpose, risks, and procedures involved were explained to all participants, who were then asked to provide written informed consent.

\section{Measurement of tongue length}

Tongue length was evaluated by measuring the MTPL in maximal volition, and the free tongue length (FTL) in resting state [16].

MPTL was measured with a transparent plastic ruler, whereas FTL was determined using a quick tongue-tie assessment tool by a blinded examiner (one physiatrist). These measurements were recorded in centimeter up to 


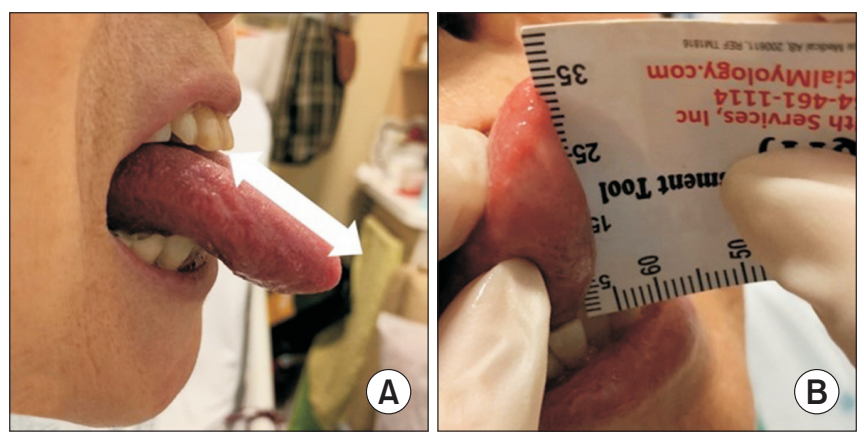

Fig. 1. Measurement of tongue length parameters: (A) MTPL and (B) FTL using quick tongue-tie assessment tool. Ratio of MTPL to FTL was calculated by dividing MTPL by FTL. MTPL, maximal tongue protrusion length; FTL, free tongue length.

one decimal place.

MTPL was defined as the distance from the tip of the tongue to the upper incisor at complete protrusion [17] (Fig. 1A). In the dangling sitting position, the patient was asked to protrude the tongue as far as possible and maintain a flat tongue. Measurement of MTPL was repeated three times with 60 seconds of rest in between the trials. The maximum and average values were then recorded.

FTL was defined as the length from the tongue-tie to the tip of the tongue in resting state, following Kotlow's free-tongue measurement [24] (Fig. 1B). The length of the fully extended tongue in the dangling sitting position was measured from the lingual frenulum to the tip.

These measurements were performed before the patient underwent the VFSS on the same day. The participants were allowed 10 minutes to familiarize themselves with the tasks before performing the measurements. The evaluation was conducted after 5 minutes to exclude the effects of learning curve, and to improve the reliability of the measurements.

Because tongue length varies from person to person, we calculated the ratio of MTPL to FTL (RMF) to reflect the difference. The inter-session reproducibility of the measurement was confirmed before the study. In this study, two rehabilitation medicine residents measured the FTL and MTPL and calculated the RMF in 10 patients to evaluate the inter-investigator consistency of tongue length measurement.

\section{VFSS protocol and interpretation}

Swallowing function was confirmed by VFSS in all pa- tients. A modified version of the Logemann protocol was used to perform the VFSS [11]. The videofluoroscopy instrument was placed lateral to the patients. First, using a spoon, patients were provided $5 \mathrm{~mL}$ of yogurt (International Dysphagia Diet Standardization Initiative [IDDSI] level 4) to swallow, followed by $4 \mathrm{~g}$ of cooked rice with barium (IDDSI level 7), and finally, $5 \mathrm{~mL}$ of water with diluted barium (IDDSI level 0). The entire procedure was recorded. The results of the VFSS were scored on the Videofluoroscopic Dysphagia Scale (VDS), Functional Oral Intake Scale (FOIS), and Penetration-Aspiration Scale (PAS) $[25,26]$. The results were analyzed by a physiatrist who was experienced in VFSS reading.

VDS is a reliable and objective predictor of dysphagia after stroke, with sensitivity and specificity of 0.91 and 0.92 , respectively [25]. It consists of 14 factors ranging from 0 to 100 . Higher scores are related to more severe dysphagia.

FOIS was scored using the recommended diet according to the VFSS results [25]. Swallowing function is graded according to the patients' dietary limitations from grade 1 (no oral feeding) to grade 7 (no limitation of diet).

The PAS is frequently used to evaluate pharyngeal dysphagia. Higher PAS scores reflect higher levels of severe pharyngeal dysphagia [26]. PAS 1 is a normal swallow with no material in the airway, whereas scores of 2-5 indicate penetration, and scores of 6-8 indicate aspiration.

\section{Statistical analysis}

Appropriate number of subjects for this research was calculated by using the method mentions in easy receiver operating characteristic (ROC): an interactive web-tool for ROC curve analysis using R language environment [27], with reference to the previous study on the relationship between tongue strength and aspiration [28].

All data were analyzed with SPSS for Windows (version 23.0; IBM Inc., Armonk, NY, USA). Raw data were used to analyze the tongue length. Descriptive statistics and tests for normality (Shapiro-Wilk test) were performed for all outcome variables.

Two sample t-tests and a Mann-Whitney U test were used to compare continuous variables between two groups (aspirator vs. non-aspirator) according to the Shapiro-Wilk test. Pearson correlation analysis and Spearman rank correlation were used to evaluate the correlation between each tongue length measurement and 
the swallowing function scale. A ROC curve was used to evaluate the sensitivity and specificity of RMF in the presence of aspiration. The intraclass correlation coefficient (ICC) was obtained based on the agreement between two examiners established using a two-way mixed model. Statistical significance was set at $\mathrm{p}<0.05$.

\section{RESULTS}

The study involved 47 post-stroke patients includ- ing 32 (68\%) diagnosed with an ischemic stroke and 15 (32\%) with intracranial hemorrhage. The length of stay for stroke in participants averages 196.98 days, and the median value was 27 days (min-max, 3-1,942). Baseline characteristics of participants are summarized in Table 1.

The participants were divided into two groups (aspirator and non-aspirator) according to the PAS score. The aspirator group included patients who scored a PAS score 6 or higher in any food material (semisolid, solid or liquid).

There was no significant difference between the two

Table 1. Participants' baseline characteristics $(n=47)$

\begin{tabular}{|c|c|c|c|}
\hline & Non-aspirator $(n=28)$ & Aspirator $(n=19)$ & p-value \\
\hline Age (yr) & $65.11 \pm 12.51$ & $69.79 \pm 12.23$ & 0.21 \\
\hline \multicolumn{4}{|l|}{ Sex } \\
\hline Male & $16(57.14)$ & $13(68.42)$ & 0.63 \\
\hline Female & $12(42.86)$ & $6(31.58)$ & \\
\hline Length of stay & $27(14.75-100.5)$ & $38(17.5-116.5)$ & 0.64 \\
\hline \multicolumn{4}{|l|}{ Stroke etiology } \\
\hline Ischemic stroke & $18(64.29)$ & $14(73.68)$ & 0.72 \\
\hline $\mathrm{ICH}$ & $10(35.71)$ & $5(26.32)$ & \\
\hline \multicolumn{4}{|l|}{ Stroke laterality } \\
\hline Right & $14(50.0)$ & $7(36.84)$ & 0.19 \\
\hline Left & $12(42.86)$ & $7(36.84)$ & \\
\hline Bilateral & $2(7.14)$ & $5(26.32)$ & \\
\hline \multicolumn{4}{|l|}{ Lesion location } \\
\hline Brainstem lesion & $9(32.14)$ & $9(47.37)$ & 0.45 \\
\hline Non-brainstem lesion & $19(67.86)$ & $10(52.63)$ & \\
\hline
\end{tabular}

Values are presented as mean \pm standard deviation or number (\%) or median (interquartile range).

$\mathrm{ICH}$, intracranial hemorrhage.

Table 2. Comparison of tongue length parameters and dysphasia scales between aspirator and non-aspirator groups

\begin{tabular}{|c|c|c|c|}
\hline & Non-aspirator $(n=28)$ & Aspirator $(n=19)$ & p-value \\
\hline \multicolumn{4}{|c|}{ Tongue length parameter } \\
\hline FTL & $2.96 \pm 0.62$ & $3.22 \pm 0.63$ & 0.16 \\
\hline MTPL & $5.38 \pm 1.24$ & $4.35 \pm 1.13$ & $0.005^{*}$ \\
\hline RMF & $1.89 \pm 0.35$ & $1.38 \pm 0.22$ & $<0.001^{*}$ \\
\hline \multicolumn{4}{|c|}{ Dysphagia scale } \\
\hline VDS & $18.32 \pm 11.84$ & $40.24 \pm 9.13$ & $<0.001^{*}$ \\
\hline FOIS & $6(6-7)$ & $2(1-4)$ & $<0.001^{*}$ \\
\hline PAS & $2(1-3)$ & $8(7-8)$ & $<0.001^{*}$ \\
\hline
\end{tabular}

Values are presented as mean \pm standard deviation or median (interquartile range).

FTL, free tongue length; MTPL, maximal tongue protrusion length; RMF, ratio of MTPL to FTL; VDS, videofluoroscopic dysphagia scale; FOIS, Functional Oral Intake Scale; PAS, Penetration-Aspiration Scale.

${ }^{*} \mathrm{p}<0.05$, Mann-Whitney U test. 
groups in baseline characteristics. The patients were divided into groups based on the presence of aspiration, as indicated by the VFSS findings. Mann-Whitney U test was used to analyze the three swallowing function scales (PAS, FOIS, and VDS) according to the normality test results. All of these scales revealed statistical differences between the two groups $(\mathrm{p}<0.001)$.

\section{FTL and the swallowing function}

FTL showed no statistical difference between aspirators and non-aspirators ( $2.96 \pm 0.62$ vs. $3.22 \pm 0.63 ; \mathrm{p}=0.16)$. Correlation analyses between the swallowing function scales also indicated no associations (VDS $\mathrm{r}=0.125, \mathrm{p}=0.41$; PAS $\rho=0.238, p=0.11$; FOIS $\rho=-0.136, p=0.36$ ) (Table 2).

\section{MTPL and swallowing function}

MTPL confirmed a significant difference between the two groups, and the non-aspirator group showed a larger value than the aspirator group (5.38 \pm 1.24 vs. $4.35 \pm 1.13$; $\mathrm{p}=0.005$ ).

MTPL was correlated with three swallowing function scales; higher MTPL was associated with lower VDS and
PAS scores, and improved FOIS scores (VDS $\mathrm{r}=-0.456$, $\mathrm{p}=0.001$; PAS $\rho=-0.475, \mathrm{p}<0.001$; FOIS $\rho=0.513, \mathrm{p}<0.001$ ) (Fig. 2).

\section{RMF and swallowing function}

RMF also established a significant difference between the two groups, with the non-aspirator group showing a larger value than the aspirator group ( $1.89 \pm 0.35$ vs. $1.38 \pm 0.22 ; \mathrm{p}<0.001)$.

Similar to MTPL, RMF was also correlated with the three swallowing function scales (VDS $\mathrm{r}=-0.663, \mathrm{p}<0.001$; PAS $\mathrm{p}=-0.772, \mathrm{p}<0.001$; FOIS $\mathrm{p}=0.717, \mathrm{p}<0.001$ ) (Fig. 3 ). Even when partial correlation coefficients were obtained with MTPL as a control variable, RMF showed a significant association with the three swallowing function scales (VDS $\mathrm{r}=-0.544, \mathrm{p}<0.001$; PAS $\rho=-0.691, \mathrm{p}<0.001$; FOIS $\rho=0.592, \mathrm{p}<0.001)$.

The cut-off value of RMF for aspiration prediction was 1.56 ; the sensitivity was $84 \%$ and specificity was $86 \%$ on the ROC curve (positive predictive value $80 \%$; negative predictive value $89 \%$; $95 \%$ confidence interval [CI], 0.810.99) (Fig. 4).
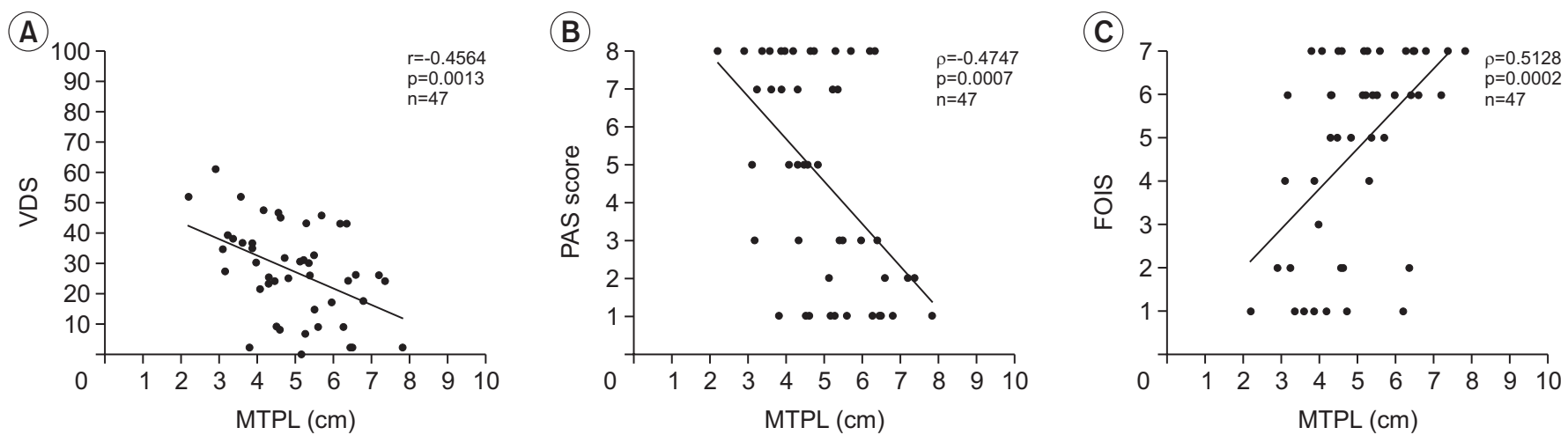

Fig. 2. Correlation between MTPL and dysphagia scales. (A) Videofluoroscopic Dysphagia Scale (VDS). (B) Penetration-Aspiration Scale (PAS) score. (C) Functional Oral Intake Scale (FOIS). MTPL, maximal tongue protrusion length.

Table 3. Inter-rater reliability of tongue length measurements

\begin{tabular}{|c|c|c|c|c|c|}
\hline & \multirow{2}{*}{ ICC } & \multicolumn{2}{|c|}{ 95\% CI } & \multirow{2}{*}{$\mathbf{F}$} & \multirow{2}{*}{ p-value } \\
\hline & & Lower & Upper & & \\
\hline FTL & 0.987 & 0.948 & 0.997 & 70.21 & $<0.001^{*}$ \\
\hline MTPL & 0.999 & 0.998 & 0.999 & $1,600.48$ & $<0.001^{*}$ \\
\hline $\mathrm{RMF}$ & 0.995 & 0.981 & 0.999 & 186.90 & $<0.001^{*}$ \\
\hline
\end{tabular}

FTL, free tongue length; MTPL, maximal tongue protrusion length; RMF, ratio of the MTPL to the FTL; ICC, intraclass correlation coefficient; $\mathrm{CI}$, confidential interval.

${ }^{*} \mathrm{p}<0.05$. 

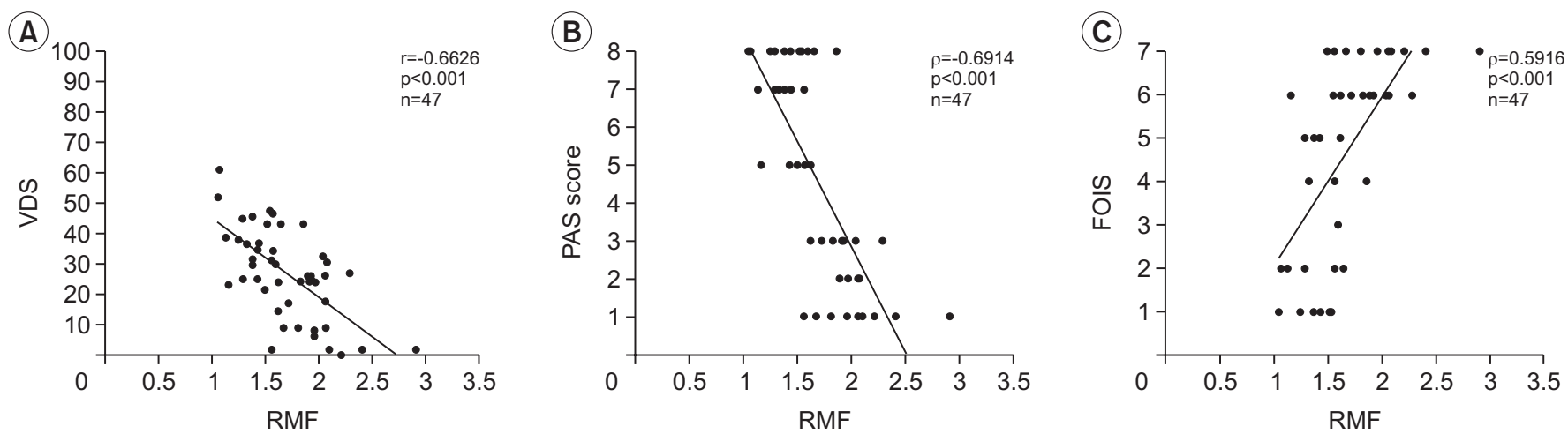

Fig. 3. Correlation between RMF and dysphagia scales. (A) Videofluoroscopic Dysphagia Scale (VDS). (B) PenetrationAspiration Scale (PAS) score. (C) Functional Oral Intake Scale (FOIS). RMF, ratio of maximal tongue protrusion length to free tongue length.

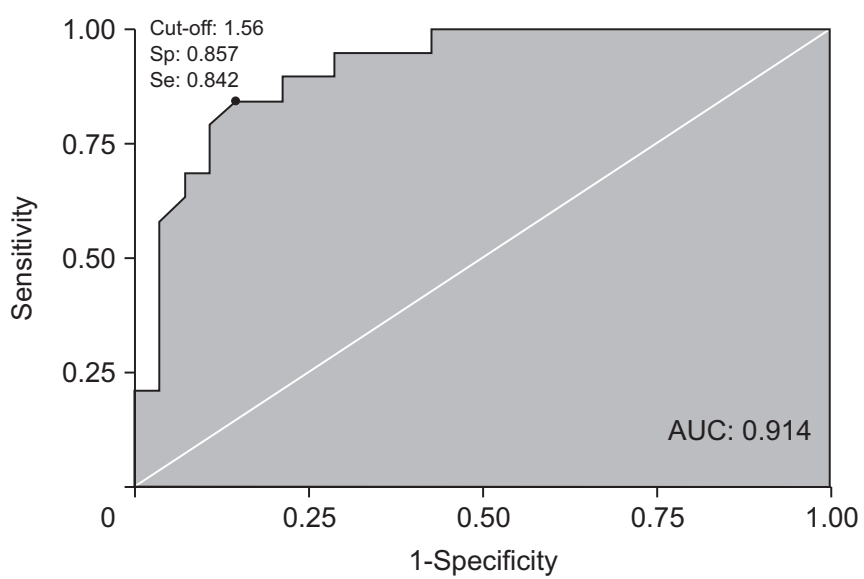

Fig. 4. Receiver operating characteristic curve of RMF for prediction of aspiration. RMF, ratio of maximal tongue protrusion length to free tongue length; AUC, area under the curve.

\section{ICC of tongue length measurements}

An ICC value of 0.9 or higher was obtained for the three tongue length measurement methods (Table 3).

\section{DISCUSSION}

This study confirmed the association between FTL, MTPL and RMF, and PSD. The smaller the MTPL and RMF, the worse was the swallowing function. There was no association between FTL and PSD.

No previous studies investigated the association between MTPL and dysphagia. MTPL is inevitably affected by the tongue length. The tongue is a soft tissue, changing its shape and length depending on its posture along with that of the mouth. Further, the person-to-person variation is large. In general, tongue length itself is not significantly related to the pharyngeal phase of the swallowing process. The tongue is anatomically divided into base of tongue (posterior one-third) and oral tongue (anterior two-thirds) depending on whether or not they are attached to the base. The swallowing function is related to the volume of the base of the tongue than to the oral tongue [29]. Therefore, this study calculated RMF to minimize the effect of the tongue itself on MTPL. The results of this study also revealed that RMF had a greater correlation with swallowing function parameters including PAS, than MTPL ( $\rho=-0.7716$ vs. $\rho=-0.4747$ ).

Tongue movement is controlled by three muscle groups: intrinsic, extrinsic and suprahyoid muscles. Intrinsic tongue muscles play a role in changing the shape of the tongue, whereas extrinsic and suprahyoid muscles control the tongue position within the oral cavity [911]. Pittman et al. [30] used electromyography (EMG) to determine the action of intrinsic and extrinsic tongue muscles during tongue protrusion in normal persons. As a result, the extrinsic tongue muscle is involved in determining the anterior-posterior tongue location, and the intrinsic tongue muscle serves as a propulsive force to ensure functioning. In the early stage of maximal tongue protrusion, most of the protrusion occurs via intrinsic and extrinsic tongue muscles, but further protrusion increases the suprahyoid muscle activity [9-11]. In the normal deglutition process, differences occur depending on the properties of the bolus; however, the tongue pressure and suprahyoid control tongue movements simultaneously from the early stage of the oral phase $[31,32]$. Taniguchi et al. [33] measured suprahyoid muscle activity 
according to tongue position in normal subjects using surface EMG and confirmed that suprahyoid activity increased under tongue protrusion. Thus, tongue movement is closely related to suprahyoid muscle activity.

MTPL is the length from the upper incisor to the tip of the tongue, and indicates the tongue length when measured from the oral cavity. It can enable the identification of the tongue location in the oral cavity. Therefore, the intrinsic and extrinsic tongue muscles together with suprahyoid muscles determine the MTPL.

FTL is the length from the frenulum insertion to the tip of the tongue. It is a widely used index in the diagnosis and classification of ankyloglossia. The shorter the FTL, the more severe is the limitation of tongue mobility [19]. FTL is used to measure the length of a part of the intrinsic tongue muscle, excluding the extrinsic tongue muscle and suprahyoid muscle. The RMF, which is the ratio obtained by dividing the MTPL by the FTL, reflects the extrinsic tongue muscle and suprahyoid muscle activity more relatively.

The relationship between MTPL and PSD is based on tongue strength and suprahyoid muscle activity.

\section{Tongue strength}

To evaluate tongue strength, many studies have measured tongue pressure generated by intrinsic and extrinsic tongue muscle activity. In a study conducted by Robbins et al. [34] involving patients with post-stroke dysphagia, an increase in tongue volume was confirmed on MRI in patients with increased maximum swallowing tongue pressure after lingual exercise.

Tongue pressure plays a role in bolus formation and transport processes in the oral phase of normal deglutition. Mid-to-median tongue pressure is an important factor in bolus transport, especially during the ingestion of semisolid foods [31]. Posterior tongue pressure also plays an important role in establishing the swallowing pressure necessary to transfer the bolus into the hypopharynx during the pharyngeal phase [35]. Previous studies have demonstrated the association between tongue pressure and oral dysphagia $[13,36]$. Tongue pressure is particularly associated with bolus formation, mastication, and oral clearance.

Kim et al. [14] reported that tongue-to-palate resistance training for 4 weeks in subacute stroke patients with dysphagia resulted insignificant improvement in both the oral and pharyngeal phases of VDS, along with an increase in tongue strength. Previous studies have revealed a relationship between MTPL and tongue pressure [16]. These studies confirmed that the longer the MTPL, the greater was the posterior tongue pressure in normal adults.

Taken together, tongue pressure represents an index of the oral and pharyngeal phases of deglutition, and is positively correlated with MTPL.

\section{Suprahyoid muscle activity}

MPTL and RMP also reflect suprahyoid muscle activity. As previously described, the suprahyoid muscle is involved in tongue protrusion. A study using surface EMG reported that the suprahyoid muscle activity is increased by the extent of tongue protrusion [16]. Among the suprahyoid muscles, the geniohyoid and mylohyoid muscles are attached to the mandible and hyoid bone. They generate hyoid bursts during normal swallowing and the progression to the pharyngeal phase. They also act in conjunction with the infrahyoid muscle to provide laryngeal elevation and regulate airway protection during swallowing.

The suprahyoid muscle is also involved in the oral phase. When ingesting semisolids or liquid, it elevates the floor of the oral cavity and facilitates bolus transport [32]. In addition, the volume of the geniohyoid muscle also affects tongue pressure [37]. Therefore, MTPL can be used to predict dysphagia based on tongue pressure and suprahyoid muscle activity.

However, MTPL is greatly affected by tongue length, which varies from person to person, suggesting limitation in setting a standardized cut-off value based on MTPL alone. Therefore, in this study, RMF was calculated. RMF has high specificity for the aspiration observed in VFSS, suggesting its role as a screening tool for PSD. According to the results of this study, at a cut-off value of 1.56 , the RMF has a sensitivity of 0.842 and a specificity of 0.857 for aspiration.

We propose advantages of RMF when compared to screening tests such as the GUSS, Johns Hopkins Hospital Brain Rescue Unit Modified 3 oz Swallow Screen, and the water swallow test, which are applied in clinical practice for PSD.

(1) The traditional bedside screening tests evaluated the presence or absence of dysphagia by monitor- 
ing symptoms for aspiration such as reflex cough, suggesting poor sensitivity for silent aspiration. In contrast, RMF can be used to evaluate tongue and suprahyoid muscle strength regardless of aspiration symptoms, and thus the risk of silent aspiration can also be evaluated.

(2) Compared with other screening tests, RMF evaluation is based on fewer items; and is a simple and user-friendly tool.

(3) Other screening tests are based on subjective criteria such as "intact," "inadequate," or "none," for each evaluated item, whereas RMF presents a clear and objective cut-off value.

In the case of the water swallow test, which has been widely used to evaluate swallowing function, the sensitivity of predicting the risk of aspiration in stroke patients was $64 \%-79 \%$, and the specificity was $61 \%-81 \%$ [37].

The relatively recent Johns Hopkins Hospital Brain Rescue Unit Modified 3 oz Swallow Screen, a screening test consisting of a water swallow test and a clinical examination, yielded a sensitivity of $46 \%$ and a specificity of $100 \%$ in stroke patients [22].

In the case of GUSS, the overall sensitivity of predicting the risk of aspiration in stroke patients was $96.5 \%$ and the specificity was $55.8 \%$; however, the sensitivity and specificity differ according to the patient's National Institutes of Health Stroke Scale (NIHSS) score (NIHSS $<5$ group, sensitivity of $71.4 \%$ and specificity of $88.8 \%$; NIHSS $>15$ group, sensitivity of $100 \%$, specificity of $20 \%$ ) [20].

The sensitivity and specificity of RMF is better than that of these commonly used screening tests.

This study has several limitations. (1) Since this study involved a heterogenous group, a larger study population and a more homogenous sample are needed. (2) In this study, the causal relation could not be confirmed due to lack of long-term observation. Therefore, subsequent studies with long-term observation are required. (3) RMF requires active cooperation of patients and is of limited value in those with cognitive impairment, and is difficult to utilize in patients with poor coordination. However, inter-rater reliability was high, so challenges associated with measurement difficulty were minimal. (4) In addition, since most stroke patients are elderly, dental defects may limit the use of MTPL when both maxillary central incisors are defective. (5) We did not evaluate the relationship between the tongue length parameters and swallowing function in a healthy population. There have been no previous studies evaluating the relationship between MTPL and swallowing function in a healthy population. Therefore, we should be careful when interpreting the results of this study.

In conclusion, MTPL and RMF were associated with the presence of dysphagia in post-stroke individuals, confirming the utility of RMF in predicting dysphagia in such patients. Clinical application of RMF requires further studies involving larger populations comprising diverse patient groups.

\section{CONFLICT OF INTEREST}

No potential conflict of interest relevant to this article was reported.

\section{ACKNOWLEDGMENTS}

This work was supported by the Soonchunhyang University Research Fund.

\section{AUTHOR CONTRIBUTION}

Conceptualization: Cho HC, Noh JS, Park JW, Choi YH, Chun SM. Methodology: Cho HC, Park JW, Noh JS, Park CW. Formal analysis: Cho HC, Choi YH, Chun SM. Project administration: Cho HC, Chun SM. Visualization: Cho HC. Writing - original draft: Cho HC, Chun SM. Writing - review and editing: Cho HC, Choi YH, Chun SM. Approval of final manuscript: all authors.

\section{REFERENCES}

1. Cohen DL, Roffe C, Beavan J, Blackett B, Fairfield CA, Hamdy S, et al. Post-stroke dysphagia: a review and design considerations for future trials. Int J Stroke 2016;11:399-411.

2. Bray BD, Smith CJ, Cloud GC, Enderby P, James M, Paley L, et al. The association between delays in screening for and assessing dysphagia after acute stroke, and the risk of stroke-associated pneumonia. J Neurol Neurosurg Psychiatry 2017;88:25-30.

3. Steinhagen V, Grossmann A, Benecke R, Walter U. Swallowing disturbance pattern relates to brain lesion location in acute stroke patients. Stroke 2009;40:1903- 
6.

4. Groher ME, Crary MA. Dysphagia: clinical management in adults and children. St. Louis, MO: Elsevier; 2015.

5. Pearson WG Jr, Langmore SE, Zumwalt AC. Evaluating the structural properties of suprahyoid muscles and their potential for moving the hyoid. Dysphagia 2011;26:345-51.

6. Beom J, Oh BM, Choi KH, Kim W, Song YJ, You DS, et al. Effect of electrical stimulation of the suprahyoid muscles in brain-injured patients with dysphagia. Dysphagia 2015;30:423-9.

7. Pearson WG Jr, Langmore SE, Yu LB, Zumwalt AC. Structural analysis of muscles elevating the hyolaryngeal complex. Dysphagia 2012;27:445-51.

8. Huckabee ML, Cannito MP. Outcomes of swallowing rehabilitation in chronic brainstem dysphagia: a retrospective evaluation. Dysphagia 1999;14:93-109.

9. Cunningham DP, Basmajian JV. Electromyography of genioglossus and geniohyoid muscles during deglutition. Anat Rec 1969;165:401-9.

10. Dinardo LA, Travers JB. Hypoglossal neural activity during ingestion and rejection in the awake rat. J Neurophysiol 1994;72:1181-91.

11. Palmer PM, Luschei ES, Jaffe D, McCulloch TM. Contributions of individual muscles to the submental surface electromyogram during swallowing. J Speech Lang Hear Res 1999;42:1378-91.

12. Shaker R, Cook IJ, Dodds WJ, Hogan WJ. Pressure-flow dynamics of the oral phase of swallowing. Dysphagia 1988;3:79-84.

13. Clark HM, Henson PA, Barber WD, Stierwalt JA, Sherrill M. Relationships among subjective and objective measures of tongue strength and oral phase swallowing impairments. Am J Speech Lang Pathol 2003;12:40-50.

14. Kim HD, Choi JB, Yoo SJ, Chang MY, Lee SW, Park JS. Tongue-to-palate resistance training improves tongue strength and oropharyngeal swallowing function in subacute stroke survivors with dysphagia. J Oral Rehabil 2017;44:59-64.

15. Hirota N, Konaka K, Ono T, Tamine K, Kondo J, Hori $\mathrm{K}$, et al. Reduced tongue pressure against the hard palate on the paralyzed side during swallowing predicts dysphagia in patients with acute stroke. Stroke 2010;41:2982-4.
16. Fujiwara S, Fujiu-Kurachi M, Hori K, Maeda Y, Ono $\mathrm{T}$. Tongue pressure production and submental surface electromyogram activities during tongue-hold swallow with different holding positions and tongue length. Dysphagia 2018;33:403-13.

17. Oh JC. Effects of tongue-hold swallows on suprahyoid muscle activation according to the relative tongue protrusion length in the elderly individuals. Dysphagia 2019;34:382-90.

18. Oh JC. Effects of tongue-hold swallows on suprahyoid muscle activation according to the relative tongue protrusion length: a preliminary study. Springerplus 2016;5:1144.

19. Lee JH, Kim HS, Yun DH, Chon J, Han YJ, Yoo SD, et al. The relationship between tongue pressure and oral dysphagia in stroke patients. Ann Rehabil Med 2016;40:620-8.

20. Warnecke T, Im S, Kaiser C, Hamacher C, Oelenberg $\mathrm{S}$, Dziewas R. Aspiration and dysphagia screening in acute stroke: the Gugging Swallowing Screen revisited. Eur J Neurol 2017;24:594-601.

21. Chen PC, Chuang CH, Leong CP, Guo SE, Hsin YJ. Systematic review and meta-analysis of the diagnostic accuracy of the water swallow test for screening aspiration in stroke patients. J Adv Nurs 2016;72:2575-86.

22. Mulheren RW, Gonzalez-Fernandez M. Swallow screen associated with airway protection and dysphagia after acute stroke. Arch Phys Med Rehabil 2019;100:1289-93.

23. O'Neill PA. Swallowing and prevention of complications. Br Med Bull 2000;56:457-65.

24. Yoon A, Zaghi S, Weitzman R, Ha S, Law CS, Guilleminault $\mathrm{C}$, et al. Toward a functional definition of ankyloglossia: validating current grading scales for lingual frenulum length and tongue mobility in 1052 subjects. Sleep Breath 2017;21:767-75.

25. Kim J, Oh BM, Kim JY, Lee GJ, Lee SA, Han TR. Validation of the videofluoroscopic dysphagia scale in various etiologies. Dysphagia 2014;29:438-43.

26. Rosenbek JC, Robbins JA, Roecker EB, Coyle JL, Wood JL. A penetration-aspiration scale. Dysphagia 1996;11:93-8.

27. Goksuluk D, Korkmaz S, Zararsiz G, Karaagaoglu AE. easyROC: an interactive web-tool for ROC curve analysis using R language environment. R J 2016;8:213-30. 28. Keskool P, Warnpeurch L, Ongard S, Pitaksurachai P, 
Nujchanart N, Kerdnoppakhun J. The relationships among objective measures of tongue strength and risk of aspiration. Siriraj Med J 2018;70:302-9.

29. Borggreven PA, Verdonck-de Leeuw I, Rinkel RN, Langendijk JA, Roos JC, David EF, et al. Swallowing after major surgery of the oral cavity or oropharynx: a prospective and longitudinal assessment of patients treated by microvascular soft tissue reconstruction. Head Neck 2007;29:638-47.

30. Pittman LJ, Bailey EF. Genioglossus and intrinsic electromyographic activities in impeded and unimpeded protrusion tasks. J Neurophysiol 2009;101:276-82.

31. Murakami K, Hori K, Minagi Y, Uehara F, Salazar SE, Ishihara S, et al. Coordination of tongue pressure production, hyoid movement, and suprahyoid muscle activity during squeezing of gels. Arch Oral Biol 2020;111:104631.

32. Taniguchi H, Tsukada T, Ootaki S, Yamada Y, Inoue M. Correspondence between food consistency and suprahyoid muscle activity, tongue pressure, and bolus transit times during the oropharyngeal phase of swal- lowing. J Appl Physiol (1985) 2008;105:791-9.

33. Oguchi H, Watanabe T, Nakamura N, Watanabe S. Influence of tongue movements on masticatory efficiency. Dent Oral Craniofac Res 2016;2:1-6.

34. Robbins J, Kays SA, Gangnon RE, Hind JA, Hewitt AL, Gentry LR, et al. The effects of lingual exercise in stroke patients with dysphagia. Arch Phys Med Rehabil 2007;88:150-8.

35. Kahrilas PJ, Logemann JA, Lin S, Ergun GA. Pharyngeal clearance during swallowing: a combined manometric and videofluoroscopic study. Gastroenterology 1992;103:128-36.

36. Pouderoux P, Kahrilas PJ. Deglutitive tongue force modulation by volition, volume, and viscosity in humans. Gastroenterology 1995;108:1418-26.

37. Mori T, Wakabayashi H, Ogawa N, Fujishima I, Oshima F, Itoda $\mathrm{M}$, et al. The mass of geniohyoid muscle is associated with maximum tongue pressure and tongue area in patients with sarcopenic dysphagia. J Nutr Health Aging 2021;25:356-60. 